\title{
The Kinetics of Charge Recombination in DNA Hairpins Controlled by Counterions
}

\author{
Gail S. Blaustein, Frederick D. Lewis, Alexander L. Burin, \\ and Rajesh Shrestha
}

Departments of Chemistry, Tulane University, New Orleans, LA 70118

and Northwestern University, Evanston, Il 60208

\begin{abstract}
The charge recombination rate in DNA hairpins is investigated. The distance dependence for the charge recombination rate between stilbene donor $\left(\mathrm{Sd}^{+}\right)$and stilbene acceptor $\left(\mathrm{Sa}^{-}\right)$linkers separated by an AT bridge has a double exponential form. We suggest that this dependence is associated with two tunneling channels distinguished by the presence or absence of the $\mathrm{Cl}^{-}$counterion bound to $\mathrm{Sd}^{+}$. Experimentbased estimates of counterion binding parameters agree within reasonable expectations. A control experiment replacing the $\mathrm{Cl}^{-}$ion with other halide ions is suggested. Counterion substitution allows modification of the charge recombination rate in either direction by orders of magnitude.
\end{abstract}

Keywords: DNA hairpin, charge transfer, counterions.

\section{Introduction}

Electronic excitation of DNA using various optical methods is important for investigating DNA structure and biological function [1. It also plays a fundamental role in a variety of DNA applications in nanotechnology which have been extensively considered during the past decade [2, [3], 4], [5]. The first timeresolved observation of charge transfer in DNA was made using stilbene capped DNA hairpins (Fig 1) 6, which are used successfully in the investigation of DNA electronic excitations and their kinetics. It is remarkable that hairpins with polyA poly-T bridges connecting stilbene acceptor (Sa) and donor (Sd) linkers (Fig 1) can possess extremely long recombination times for the charge separated state $\mathrm{Sa}^{-}(\mathrm{AT})_{n} \mathrm{Sd}^{+}$following Sa photoexcitation. Increasing the number of AT pairs forming the bridge from $n=1$ to $n=7$ reduces the charge recombination rate by eight orders of magnitude [7]. This interesting property of stilbene capped DNA hairpins has potential for a variety of applications involving charge separation such as solar cells [8].

Therefore, it is important to understand mechanisms of charge recombination in DNA hairpins and investigate possible ways to control this process. This requires understanding distance dependence of the charge transfer rate (1).

G. Allen et al. (Eds.): ICCS 2009, Part II, LNCS 5545, pp. 189-196, 2009.

(C) Springer-Verlag Berlin Heidelberg 2009 


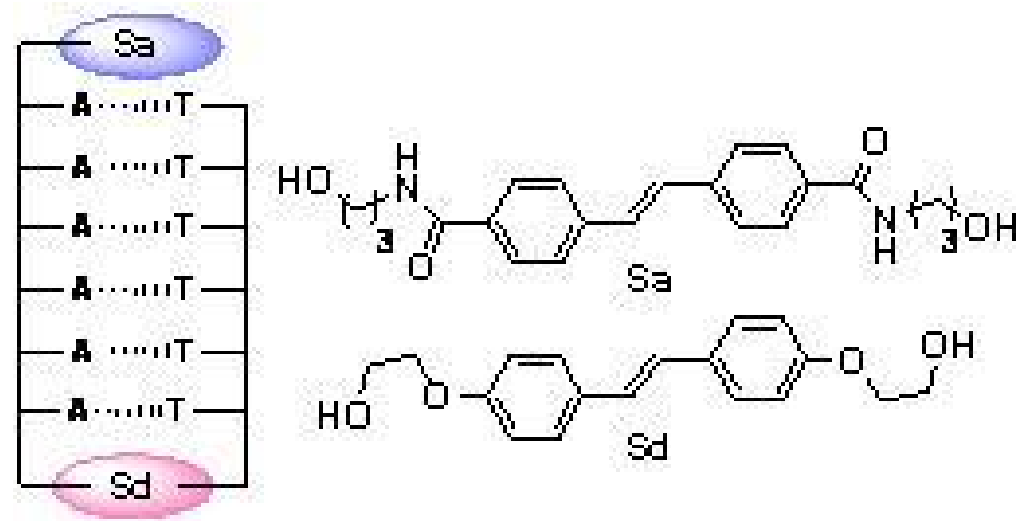

Fig. 1. A hairpin with six AT base pairs and the stilbene linkers

\section{Kinetics Model}

In this paper, the charge recombination rate in poly-A poly-T DNA hairpins [6] of lengths $n=1$ to $n=7$ is investigated. It is shown that the charge recombination rate for AT bridges as a function of distance $r$ between $\mathrm{Sd}^{+}$and $\mathrm{Sa}^{-}$has the double exponential form

$$
k(r)=k_{0}\left[\exp \left(-\beta_{1} r\right)+C \exp \left(-\beta_{2} r\right)\right] .
$$

In our investigation, we consider the hairpin in its environment of a dilute aqueous solution of $\mathrm{NaCl}$. In our model, we propose that this double exponential behavior is associated with two distinguishable charge separated states for the hairpin. State 1 is the state where $\mathrm{Cl}^{-}$is attached to $\mathrm{Sd}^{+}$and state 2 is the state where $\mathrm{Sd}^{+}$is isolated, that is where $\mathrm{Sd}^{+}$only has water molecules nearby. Let $\Delta$ denote the energy difference between states 1 and 2 , with the phase volume $\Omega>>1$ for state 2 , as $\mathrm{Cl}^{-}$is dilute in water. Let $P_{1}$ and $P_{2}$ represent the probabilities of the hairpin occupying states 1 and 2 respectively. Consider time $t=0$ as the time of hole arrival at Sd.

The time evolution of these two probabilities is determined by two processes including hole recombination with rates $k_{1}$ and $k_{2}$ from states 1 and 2 respectively and fluctuation of the system between states 1 and 2 with rates $w_{12}$ and $w_{21}$ for transitions $1 \rightarrow 2$ and $2 \rightarrow 1$ respectively. Rates $w_{12}$ and $w_{21}$ characterize the process of counterion dissociation and association respectively. For thermal equilibrium probabilities $P_{1}^{*}$ and $P_{2}^{*}$, they must satisfy the detailed balance principle

$$
P_{1}^{*} w_{12}=P_{2}^{*} w_{21} .
$$

As $P_{2}$ is the probability that the $\mathrm{Cl}^{-}$counterion is not bound to $\mathrm{Sd}^{+}$, we can express the ratio of probabilities $P_{2} / P_{1}$ as $C$, where

$$
C=\Omega \exp \left(-\Delta / k_{B} T\right),
$$


so that

$$
w_{12}=C w_{21} \text {. }
$$

The time dependence of $P_{1}$ and $P_{2}$ is determined by the rate equations

$$
\begin{aligned}
& \frac{d P_{1}}{d t}=-k_{1} P_{1}-w_{12} P_{1}+w_{21} P_{2} \\
& \frac{d P_{2}}{d t}=-k_{2} P_{2}-w_{21} P_{2}+w_{12} P_{1} .
\end{aligned}
$$

An experimentally observable parameter is survival probability $P(t)=P_{1}(t)+$ $P_{2}(t)$. Its time decay characterizes the recombination rate.

The solution to (5) is

$$
\left(\begin{array}{l}
P_{1}(t) \\
P_{2}(t)
\end{array}\right)=f_{1}\left(\begin{array}{l}
1 \\
a
\end{array}\right) \exp \left(-\lambda_{1} t\right)+f_{2}\left(\begin{array}{l}
1 \\
b
\end{array}\right) \exp \left(-\lambda_{2} t\right)
$$

where

$$
\begin{aligned}
& a=w_{12} /\left(-\lambda_{1}+k_{2}+w_{21}\right) \\
& b=w_{12} /\left(-\lambda_{2}+k_{2}+w_{21}\right) .
\end{aligned}
$$

The decay rates $\lambda_{1}$ and $\lambda_{2}$ are the solutions to the characteristic equation for (5) and constants $f_{1}$ and $f_{2}$ are determined by initial conditions $P_{1}(0)$ and $P_{2}(0)$, where the survival probability $P(0)=P_{1}(0)+P_{2}(0)=1 . \lambda_{1}, \lambda_{2}, f_{1}$ and $f_{2}$ are determined to be

$$
\begin{aligned}
& \lambda_{1}=\frac{w_{12}+w_{21}+k_{1}+k_{2}}{2}-\sqrt{\frac{\left(-w_{12}+w_{21}-k_{1}+k_{2}\right)^{2}}{4}+w_{21} w_{12}} \\
& \lambda_{2}=\frac{w_{12}+w_{21}+k_{1}+k_{2}}{2}+\sqrt{\frac{\left(-w_{12}+w_{21}-k_{1}+k_{2}\right)^{2}}{4}+w_{21} w_{12}}
\end{aligned}
$$

and

$$
\begin{aligned}
& f_{1}=-\frac{P_{2}(0)-a P_{1}(0)}{a-b} \\
& f_{2}=\frac{P_{2}(0)-b P_{1}(0)}{a-b} .
\end{aligned}
$$

Now we assume recombination is slow; that is $k_{1}, k_{2}<w_{12}+w_{21}$. Indeed, using the diffusion rate $D \approx 10^{-5} \mathrm{~cm}^{2} / \mathrm{s}[9$ and assuming that a counterion moves by random jumps of $1 \AA$, one can estimate that during the minimum recombination time of 10 ns the counterion jumps approximately 1000 times, which should be sufficient for the ion to access the bound state near the $\mathrm{Sd}^{+}$group as $\left[\mathrm{Cl}^{-}\right] \sim 0.1 \mathrm{M}$ (about 1 chloride ion per 500 water molecules). Thus the equilibration rate $w=$ $w_{12}+w_{21}$ should exceed the hole recombination rates. 
Using our assumption, we can approximate the decay rates $\lambda_{1,2}$ via Taylor expansion by the lowest order in $k_{1,2} / w$ as

$$
\begin{aligned}
& \lambda_{1} \approx \frac{w_{12} k_{2}+w_{21} k_{1}}{w_{12}+w_{21}} \\
& \lambda_{2} \approx w_{12}+w_{21} .
\end{aligned}
$$

With this approximation, the solution of (5) reads

$$
\begin{array}{r}
\left(\begin{array}{l}
P_{1}(t) \\
P_{2}(t)
\end{array}\right)=\left(P_{1}(0)+P_{2}(0)\right)\left(\begin{array}{l}
w_{21} /\left(w_{12}+w_{21}\right) \\
w_{12} /\left(w_{12}+w_{21}\right)
\end{array}\right) \exp \left(-\lambda_{1} t\right) \\
+\frac{P_{1}(0) w_{12}-P_{2}(0) w_{21}}{w_{12}+w_{21}}\left(\begin{array}{c}
1 \\
-1
\end{array}\right) \exp \left(-\lambda_{2} t\right) .
\end{array}
$$

It follows that the derived survival probability can be expressed as

$$
P(t)=P_{1}(t)+P_{2}(t) \approx \exp (-\lambda t) .
$$

Thus the observed decay rate can be well approximated by

$$
\lambda_{1}=\frac{w_{12} k_{2}+w_{21} k_{1}}{w_{12}+w_{21}} .
$$

Taking $\lambda_{1}=k$, (11) follows directly from (4) and (10) as the thermodynamic average of the two state recombination rates. $\beta_{1}$ and $\beta_{2}$ are taken to be the experimentally derived values $0.97 \AA^{-1}$ and $0.42 \AA^{-1}$ respectively [7].

A Hückel model is used to describe charge tunneling from $\mathrm{Sd}^{+}$to $\mathrm{Sa}^{-}[10,[11$. This model should be reasonably relevant in the tunneling regime despite polaron formation and possible environmental fluctuations which can reduce the energy barrier. We can then determine the electron overlap integral $b$ for adjacent AT base pairs. We assume that the thermal equilibrium of the counterion and the $\mathrm{Sd}^{+}$ion is established before recombination begins. This is justified by the large diffusion rate of counterions in water: $D \approx 10^{-5} \mathrm{~cm}^{2} / \mathrm{s}$ (see [9]).

According to our model, (3) is the probability that counterion $\mathrm{Cl}^{-}$is not bound to the $\mathrm{Sd}^{+}$group, where $\Delta$ is the binding energy and $k_{B} T=0.026 \mathrm{eV}$ is the thermal energy at room temperature. Experiments [7] were performed in a $0.1 \mathrm{M}$ aqueous solution of $\mathrm{NaCl}$. The prefactor $\Omega$ can be estimated as the ratio of water molecules per chloride ion, so that $\Omega \sim 556$. The error of this estimate is assumed to be less than an order of magnitude so we can consider $10^{2}<\Omega<10^{3}$. Lower and upper boundaries for $\Omega$ will be used to estimate the accuracy of our estimate of the binding energy $\Delta$. Solving (3) with the probability $C$ taken from experimental data [7] (see (10), one finds

$$
0.36 \mathrm{eV}<\Delta<0.42 \mathrm{eV} .
$$

This estimate is within the range of typical counterion binding energies [12, 13 .

In the next step, a relationship is derived between energies $E_{1}$ and $E_{2}$ of the $\mathrm{Sd}^{+}$linker in the presence and absence of $\mathrm{Cl}^{-}$respectively, and corresponding 


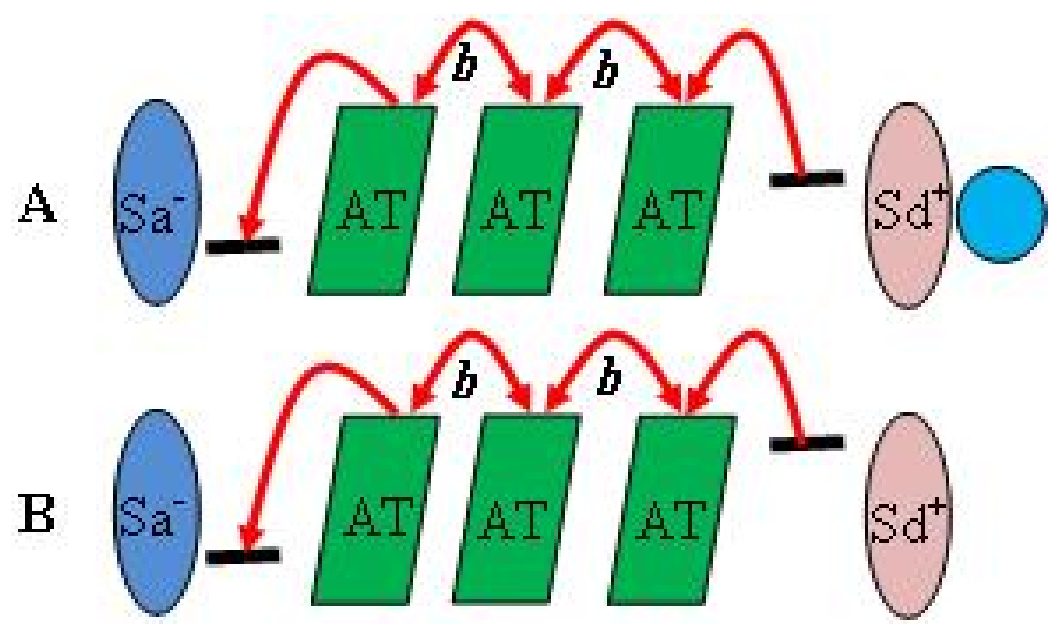

Fig. 2. Stilbene capped hairpin; charge recombination channels

tunneling exponents $\beta_{1}$ and $\beta_{2}$ in (1). The energy of an isolated (AT) ${ }^{+}$is set to zero. $E_{1}<E_{2}$ implies that

$$
E_{2}=E_{1}+\Delta
$$

One can establish a clear relationship between energy and the tunneling exponent. This relationship involves the electron transfer integral $b$ responsible for charge tunneling between adjacent AT base pairs, shown in Fig 2 It can be written as 10

$$
2 b \cosh \left(\beta_{i} a / 2\right)=E_{i}, i=1,2,
$$

where $a=3.4 \AA$ As the distance between adjacent base pairs.

This relationship can be derived as follows. The tunneling rate through $n$ AT pairs (cf. (1)) is determined by the exponential tail of the positive charge wave function $k_{n} \sim \psi_{n}^{2}$. Here $\psi_{n}^{2}$ is the probability of finding the charge at the $n$th base pair. The wavefunction of the charge under the barrier decreases exponentially with the base number as $\psi_{n} \sim \exp (-\kappa n)$, while the charge transfer rate decreases exponentially with the bridge length as $\exp (-\beta a n)$. Comparing the two exponents, we have $\kappa=\beta a / 2$. Equation (16) results from the discrete Schrödinger equation [10]

$$
E \psi_{n}=-b\left(\psi_{n-1}+\psi_{n+1}\right)
$$

if the exponential $n$ dependence for $\psi_{n}$ is assumed as above $\left(\psi_{n} \sim \exp (-\kappa n)\right)$.

Using (14), (15) and (16), one can evaluate the electron transfer integral and the energies of $\mathrm{Sd}^{+}$in the absence or presence of $\mathrm{Cl}^{-}$as $b=0.13 \pm 0.01 \mathrm{eV}, E_{1}=$ $0.73 \pm 0.06 \mathrm{eV}$ and $E_{2}=0.34 \pm 0.03 \mathrm{eV}$. It is remarkable that the estimate for the electron transfer integral $b$ agrees very well with the calculations of Voityuk and coworkers 14] for the coupling of adjacent thymine bases, but differs from the estimate for intrastrand coupling of adjacent adenine bases by a factor of 3 . 
In principle, based on the ionization potentials of DNA bases calculated in a vacuum [1415|16], one should expect that the hole tunnels through the adjacent adenine bases rather than the thymine bases because the adenine ionization potential is lower by about $1 \mathrm{eV}$. However, the real situation is complicated by the dissolution of DNA in water, which can affect ionization potential. Furthermore, tunneling is also very sensitive to bridge fluctuations [17]. The analysis of other experimental data for charge transfer through AT bridges of various lengths [18] can also be successful when only assuming electron transfer integrals to exceed ab initio estimates for them.

It is not quite clear to us as to why there is no observation of thermally activated recombination for $(\mathrm{AT})_{n}$ bridges up to $n=7$ in contrast to Ref. [18. A possible explanation of this behavior is edge effect, which does not affect tunneling, but can be crucially important for thermally activated transport. For instance, the energy of the $\mathrm{AT}^{+}$state of an AT pair adjacent to an Sd linker can be larger than the energy of other AT pairs due to its electrostatic interaction with Sd. This difference can increase the activation energy of the first hopping step thus suppressing the hopping channel.

Another reason can be the difference of charge recombination [7] with charge shift reactions [15. For example, the investigation of charge recombination in DNA hairpins using naphthaldimide and phenothiazine as acceptor and donor separated by various AT bridges [19] exhibits behavior similar to Ref.[7. Indeed, a tunneling exponent $\beta=0.40 \AA^{-1}$ was reported for charge recombination across 4-8 AT base pairs. The small preexponential factor [19] $\left(10^{8} \mathrm{~s}^{-1}\right)$ compared to $k_{0}$ in (11) can be due to the fact that charge recombination for $4-8$ base pairs also occurs without associated counterions.

\section{Discussion}

Our model can be verified in a number of ways. One way is to change the $\mathrm{NaCl}$ concentration. According to (11), the reduction of $\mathrm{NaCl}$ concentration by a factor of ten will increase the recombination rate tenfold for long AT bridges $(n>4)$.

A more interesting experiment is to replace chloride ions with other counterions, for instance $\mathrm{F}^{-}, \mathrm{Br}^{-}$or $\mathrm{I}^{-}$. The binding energy should decrease with increasing the ionic radius. Crude estimates in Table1 were made assuming that the binding energy $\Delta_{X}$ of counterion $X$ is inversely proportional to ionic radius. This estimate ignores the contribution of water to binding energy and thus underestimates possible changes in recombination rates for different counterions. We used the same values for the electron transfer integral $b$, energy $E_{2}$ of the $\mathrm{Sd}^{+}$state in the absence of a counterion (and, consequently, exponent $\beta_{2}$ ) and the phase volume factor $\Omega$. The last column in Table 1 shows the recombination rate for the longest bridge of seven AT pairs. For the smallest ion $\mathrm{F}^{-}$, the recombination time is as long as $3.33 \mathrm{~s}$.

In order to substantiate our hypothesis about counterion attachment to the Sd group and the ionic radius effect on counterion binding energy, we performed very preliminary calculations of binding energies for various halide anions with 
Table 1. Approximate parameters of recombination rates controlled by different counterions (see Eq.(1)), $\beta_{2}=0.42 \AA^{-1}$

\begin{tabular}{|c|c|c|c|c|c|}
\hline$X$ & DFT Energy $(\mathrm{eV})$ & $\Delta_{X}(\mathrm{eV})$ & $\beta_{1}\left(\AA^{-1}\right)$ & $C$ & $k_{7}\left(\mathrm{~s}^{-1}\right)$ \\
\hline $\mathrm{F}$ & 7.55 & 0.56 & 1.14 & $2.71 \times 10^{-7}$ & 0.3 \\
\hline $\mathrm{Cl}$ & 4.08 & 0.41 & 1.03 & $8.02 \times 10^{-5}$ & 88 \\
\hline $\mathrm{Br}$ & 4.42 & 0.38 & 1.01 & $2.68 \times 10^{-4}$ & 293 \\
\hline $\mathrm{I}$ & 3.88 & 0.34 & 0.97 & $1.31 \times 10^{-3}$ & 1436 \\
\hline
\end{tabular}

the $\mathrm{Sd}^{+}$group as shown in Table 1. Calculations were performed in a vacuum; therefore, all energies are overestimated. Calculations in an aqueous solution are currently in progress. The calculations were performed using density functional theory and the B3LYP/3-21G basis set. It is obvious that energies differ from our expectations by a factor of 10 which is a consequence of the absence of Coulomb interactions with water. However, the data trend has a reasonable correlation with our expectation. Moreover, the modeled binding energy of $\mathrm{F}^{-}$ ion is greater than for $\mathrm{Cl}^{-}$as compared to our expectations for these ions which leads us to expect that the charge recombination rate should be even slower than we predicted compared to the present case of $\mathrm{Cl}^{-}$ions.

It may seem odd that the binding energy for $\mathrm{Br}^{-}$is slightly larger than that for $\mathrm{Cl}^{-}$. This anomaly in the trend of decreasing binding energy of the system with increasing ionic radius of the halide counterions can possibly be understood when considering their electron affinities. We see that the electron affinity of the chloride ion is larger than for the other halide ions. The partial charge in chloride, approximately $-0.405 \mathrm{C}$, is twice as high than for other halides; therefore, it has a minimum covalent bond strength which reduces the binding energy compared to our expectations. A similar trend was reported in [20].

\section{Conclusion}

It is shown that the complicated double exponential recombination kinetics in DNA hairpins can be interpreted assuming that this process is controlled by the binding of a counterion. Key experiments are suggested to verify our theory and to control the recombination process by varying counterions.

Acknowledgments. This work is supported by the NSF CRC Program Grant No. 0628092. GSB acknowledges the support of the Department of Defense Science, Mathematics and Research for Transformation (SMART) Scholarship Program. Authors acknowledge Russ Schmehl for fruitful discussions.

\section{References}

1. Tinoco Jr., I.: Hypochromism in polynucleotides. J. Am. Chem. Soc. 82, 4785-4790 (1960)

2. Braun, E., Eichen, Y., Sivan, U., Ben-Yoseph, G.: DNA-templated assembly and electrode attachment of a conducting silver wire. Nature 391, 775-778 (1998) 
3. Meggers, E., Michel-Beyerle, M.E., Giese, B.: Sequence Dependent Long Range Hole Transport in DNA. J. Am. Chem. Soc. 120, 12950-12955 (1998)

4. Henderson, P.T., Jones, D., Hampikian, G., Kan, Y., Schuster, G.B.: Long-distance charge transport in duplex DNA: The phonon-assisted polaron-like hopping mechanism. Proc. Natl. Acad. Sci. U.S.A. 96, 8353-8358 (1999)

5. Steckl, A.J.: DNA - a new material for photonics. Nature Photonics 1, 3 (2007)

6. Lewis, F.D., Wu, T., Zhang, Y., Letsinger, R.L., Greenfield, S.R., Wasielewski, M.R.: Distance-Dependent Electron Transfer in DNA Hairpins. Science 277, 673676 (1997)

7. Lewis, F.D., Zhu, H., Daublain, P., Fiebig, T., Raytchev, M., Wang, Q., Shafirovich, V.: Crossover from superexchange to hopping as the mechanism for photoinduced charge transfer in DNA hairpin conjugates. J. Am. Chem. Soc. 128, 791-800 (2006)

8. Granstrom, M., Petritsch, K., Arias, A.C., Lux, A., Andersson, M.R., Friend, R.H.: Laminated fabrication of polymeric photovoltaic diodes. Nature 395, 257260 (1998)

9. Berg, R.: Random Walks in Biology. Princeton University Press, Princeton (1983)

10. Berlin, Y.A., Burin, A.L., Ratner, M.A.: On the Long Range Charge Transfer in DNA. Chem. Phys. 275, 61-74 (2002)

11. Berlin, Y.A., Burin, A.L., Ratner, M.A.: Charge Hopping in DNA. J. Am. Chem. Soc. 123, 260-268 (2001)

12. Angelini, T.E., Liang, H., Wriggers, W., Wong, G.: Like-charge attraction between polyelectrolytes induced by counterion charge density waves. Proc. Natl. Acad. Sci. 100, 8634-8637 (2003)

13. Naghizadeh, J.: Counterion binding in polyelectrolyte theory. In: Bennemann, K.H., Brouers, F., Quitmann, D. (eds.) Euro-Par 1982. LNP, vol. 172, pp. 242 246. Springer, New York (1982)

14. Voityuk, A.A., Rösch, N., Bixon, M., Jortner, J.: Electronic coupling for charge transfer and transport in DNA. J. Phys. Chem. 104, 9740-9745 (2000)

15. Siriwong, K., Voityuk, A.A., Newton, M.D., Rösch, N.J.: Estimate of the Reorganization Energy for Charge Transfer in DNA. Phys. Chem. B 107, 2595-2601 (2003)

16. Sugiyama, H., Saito, I.: Theoretical Studies of GG-Specific Photocleavage of DNA via Electron Transfer: Significant Lowering of Ionization Potential and 5'Localization of HOMO of Stacked GG Bases in B-Form DNA. J. Am. Chem. Soc. 118, 7063-7068 (1996)

17. Troisi, A., Orlandi, G.: The hole transfer in DNA: calculation of electron coupling between close bases. Chem. Phys. Lett. 344, 509-518 (2001)

18. Giese, B., Amaudrut, J., Köhler, A.K., Spormann, M., Wessely, S.: Direct observation of hole transfer through DNA by hopping between adenine bases and by tunnelling. Nature 412, 318-320 (2001)

19. Takada, T., Kawai, K., Cai, X., Sugimoto, A., Fujitsuka, M., Majima, T.: Charge Separation in DNA via Consecutive Adenine Hopping. J. Am. Chem. Soc. 126, 1125-1129 (2004)

20. Ríos, H., Gamboa, C., Ternero, G.: Counterion binding to cationic polyelectrolytes in aqueous solution. Journal of Polymer Science B 29, 805-809 (1991) 